\title{
Gestión directiva y el trabajo con la comunidad durante la emergencia sanitaria por el COVID-19
}

Executive management and work with the community during the health emergency due to COVID-19

Gestão executiva e trabalho com a comunidade durante a emergência sanitária decorrente do COVID-19

Ruth Giovanna Ruesta Quiroz

rruestaq@ucvvirtual.edu.pe

https://orcid.org/0000-0002-9848-2460

Universidad César Vallejo, Lima-Perú

\author{
Cindy Victoria Gejaño Ramos \\ cgejano@ucvvirtual.edu.pe \\ https://orcid.org/0000-0001-6233-3626 \\ Universidad César Vallejo, Lima-Perú
}

\author{
Carlos Sixto Vega Vilca \\ cvegacs@ucv.edu.pe \\ https://orcid.org/0000-0002-2755-8819 \\ Universidad César Vallejo, Lima-Perú
}

Artículo recibido 25 de agosto 2021, arbitrado y aceptado 8 de noviembre 2021 y publicado 28 de enero 2022

\section{RESUMEN}

El año 2020 en Perú se declaró la emergencia sanitaria por el COVID-19, esta coyuntura exigió que desde la gestión directiva se implementar acciones para garantizar la continuidad de la educación en colaboración con la comunidad, conformada por los padres de familia, por esta razón se formuló como objetivo explorar desde la literatura académica la gestión directiva y el trabajo con la comunidad durante la emergencia sanitaria por el COVID-19, se empleó el enfoque cualitativo de revisión bibliográfica, de la revisión de la literatura se concluyó que, las dificultadas en Perú fueron similares a las de otros países, en los cuales desde la dirección se debieron generar espacios de conexión, también se evidenció afecciones emocionales en los padres, quienes tuvieron dificultades en ayudar a sus hijos en esta nueva normalidad.

Palabras clave: Comunidad; COVID-19; Emociones; Gestión directiva y pandemia

\section{ABSTRACT}

In 2020 in Peru, the health emergency was declared due to COVID-19, this situation demanded that the directive management implement actions to guarantee the continuity of education in collaboration with the community, made up of parents, by this the reason was formulated as an objective to explore directive management and work with the community during the health emergency due to COVID-19 from the academic literature, the qualitative approach of bibliographic review was used, from the literature review it was concluded that, the Difficulties in Peru were very similar to those in other countries, in which the leadership had to create spaces for connection, emotional affections were also evidenced in the parents, who had difficulties in helping their children in this new normal.

Key words: Community; COVID-19; Emotions; Directive management and pandemic

\section{RESUMO}

No ano de 2020 no Peru foi declarada a emergência sanitária devido ao COVID-19, esta situação exigia que a gestão diretiva implementasse ações para garantir a continuidade da educação em colaboração com a comunidade, composta por pais, por isso foi formulada como um objetivo explorar a gestão diretiva e o trabalho com a comunidade durante a emergência sanitária devido ao COVID-19 a partir da literatura acadêmica, utilizou-se a abordagem qualitativa de revisão bibliográfica, a partir da revisão da literatura concluiu-se que as dificuldades no Peru eram muito semelhantes. Para as de outros países, em que as lideranças tiveram que criar espaços de conexão, os afetos emocionais também foram evidenciados nos pais, que tinham dificuldade em ajudar os filhos nesse novo normal.

Palavras-chave: Comunidade; COVID-19; Emoções; Gestão diretiva e pandemia 


\section{INTRODUCCIÓN}

El año 2020 a nivel mundial se expandió el virus del COVID-19, esto originó que gran parte de los países del mundo, debido a la fácil trasmisión y las consecuencias en la salud, decidieran establecer medidas sanitarias, como fue el confinamiento obligatorio o cuarentena; esta situación de inmovilidad obligatorio determinó que las actividades que habitualmente se realizaban cambiaran, por esta razón en Perú el presidente para esa fecha Martín Vizcarra en el mes de marzo decidiera suspender las clases en las instituciones de gestión privada, que ya habían empezado, postergar el inicio del año escolar de las instituciones de gestión pública hasta el mes de abril del 2020 y declarar el establecimiento del sistema de educación remota o a distancia en Educación Básica Regular (EBR) y Educación Básica Alternativa (EBA), emitiendo normativas para regularla como fue la $\mathrm{RV} \mathrm{N} 093$ “Orientaciones pedagógicas para el servicio educativo de Educación Básica durante el año 2020 en el marco de la emergencia sanitaria por el Coronavirus COVID-19”. ( MINEDU, 2020). Los responsables de dicha implementación fueron a mano del personal directivo de los distintos planteles educativos.

Para lo cual es importante destacar que la gestión directiva consiste en la labor que desempeñan los directivos, los cuales deben contar con competencia como es el conocimiento de su función y aptitudes, que le permita realizar una buena gestión en su institución. Para Rodríguez (2016) citado por López et al., (2019) la gestión son las acciones sistemáticas y organizadas que permiten cumplir los objetivos, los cuales deberán contribuir a mejorar el servicio que brinda la escuela y contribuir para la mejora de la calidad en tal sentido alcanzar la eficacia y eficiencia (Gonzales-Sánchez et al., 2021).

De acuerdo a Pérez (2014) con el afán de brindar precisiones de la gestión escolar a partir de los años 80 América Latina, ha tratado de alejarse de los principios teóricos de la gestión educativa bajo la orientación del campo administrativo; esto con la finalidad de darle un campo de acción más amplio que implique el trabajo conjunto de la escuela y políticas educativas que fortalezcan su labor. Por otro lado, Acevedo et al., (2017) emplearon el terminó de gestión efectiva, en la cual desde el liderazgo directivo se implementan acciones que permitan fortalecer los procesos de enseñanza y aprendizaje de los docentes, los cuales se evidenciaran en los logros académicos de los estudiantes. En tal sentido será importante el acompañamiento que menciona Carrasco et al., (2017) porque mejora el desempeño laboral de los docentes, para mejorar el acercamiento los directivos también es necesario el dominio de la competencias socioemocionales demostrar empatía, escucha activa y regulación de emociones, las cuales han sido indispensables en el contexto de la emergencia sanitaria (GonzalesSánchez et al., 2021).

En Perú las funciones del director se encuentran normadas en el Marco del Desempeño Directivo (MINEDU, 2014) en donde se menciona que además es inherente a su función debe cumplir los Compromisos de Gestión Escolar, los cuales favorecen al buen desempeño de la escuela y fortalecer las experiencias de aprendizaje de los estudiantes, de forma integrada, si la comunidad educativa detecta dificultades, se deben implementar planes de mejora, los cuales se incorporan a los documentos de gestión que utiliza la institución. 
En el contexto de la emergencia sanitaria fue publicada la "Guía para la gestión escolar en II.EE. y programas educativos de Educación Básica"(MINEDU, 2021b), de la cual se destacó la labor del directivo desde la gestión. Está se divide en gestión estratégica, contempla visón, misión y metas institucionales, después se ubica la gestión administrativa involucra condiciones de la operatividad en brindar el servicio educativo, en tercer lugar incluye la gestión pedagógica donde se contempla los aspectos relacionados a los docentes y rendimiento académico de los estudiantes; por último el cuarto aspecto de la gestión contempla la gestión comunitaria, direccionada en atender a los padres de familia, asimismo, generar alianzas estratégicas en la localidad.

El segundo aspecto en abordar fue el trabajo con la comunidad, desde la gestión directiva implica la atención a los padres de familia, fortalecer las condiciones necesarias para que se desarrolle un trabajo integrado, a favor de los estudiantes. En el contexto de la emergencia sanitaria se debió generar espacios de conexión con los padres de familia, los cuales facilitaran la comunicación, involucradlos en la formación académica de sus hijos, tanto en la prespecialidad como en la educación a distancia, el nexo entre la escuela y la escuela fueron los tutores.

En Perú al implantarse el nuevo sistema educativo en EBR y EBA, surgieron conflictos con los padres, los cuales cuestionaron, si esta forma de enseñanza era la adecuada o podría ser de calidad; en las instituciones de gestión privada la situación resultó más complicada (Gonzales-Sánchez et al., 2021) los reclamos de los padres fueron múltiples, principalmente la exigencia de bajar las pensiones debido a que la infraestructura no era utilizada.
Sin embargo, tanto en la escuela pública y privada, se evidenciaron las brechas digitales (Gómez, y Escobar, 2021) principalmente en la zonas rurales, que dificultaron la comunicación sincrónica y asincrónica; por otro lado, la Defensoría del Pueblo (2020), demostró que estas limitaciones ya existían; pero poco se había avanzado en mejorar esta situación; estas limitaciones del acceso no fueron exclusivas en Perú, se presentaron en gran parte de los países (CEPAL-UNESCO, 2020).

Otro aspecto del trabajo comunitario, desde la gestión directiva se deben generar alianzas con los otros actores de la comunidad, como son la municipalidad, áreas de salud, la Defensoría Municipal del Niño y del Adolescente (DEMUNA), Comisaría; etc. Debido a que, los estudiantes desde la escuela deben cumplir un rol dinámico y activo de participación cívica, empoderamiento de sus derechos y práctica de valores, preparándolos para ser futiros ciudadanos.

Por causa de la pandemia, se debieron establecer nuevas formas de conexión, con los aliados estratégicos, desde el Ministerio de Salud (MINSA) se realizaron Webinar o videoconferencias principalmente en el año 2020 con temas relacionados al COVID-19, conforme avanzó el progreso de la emergencia sanitaria, se fortalecieron los temas emocionales, hábitos de alimenticos y actividad física. El confinamiento o cuarentena, en el cual las familias tuvieron que compartir más tiempo juntos, originó situaciones de violencia (Lozano-Vargas, 2020) afectando severamente la salud mental, por esta razón la DEMUNA como aliado junto a la Comisaría, ejecutaron acciones de sensibilización de protección al menor. Por otro lado, las municipalidades se convirtieron en aliados 
de los representantes de las distintas Unidad de Gestión Local (UGEL) para buscar a los estudiantes que abandonaron la educación y ser recuperados. De acuerdo a lo antes expuesto, se formuló como objetivo de estudio explorar desde la literatura académica la gestión directiva y el trabajo con la comunidad durante la emergencia sanitaria por el COVID-19; este estudio se consideró importante porque permite exponer a la comunidad académica las dificultades, que afrontaron los directores en este contexto y las complicaciones con los padres de familia como parte de la comunidad fueron similares a lo que afrontaron otros países.

\section{METODOLOGÍA}

En esta investigación se empleó el enfoque cualitativo de revisión bibliográfica, para lo cual se consultaron las bases de datos: SCOPUS, Web of Science (WOS). ERIC Y SCIELO, se tomó como referencia 14 artículos científicos. También se emplearon resoluciones emitidas por el Ministerio de Educación de Perú, emitidos en el contexto de la emergencia sanitaria e informes de la Defensoría del Pueblo, para la selección de los artículos científicos se tomó en consideración la fecha de publicación de las investigaciones, que fueran del año 2020 y 2021, además que estuvieran elaborados en el contexto de la emergencia sanitaria y desarrollados en escuelas. Se desestimó las investigaciones, que a pesar de tener coincidencias con los temas hubieran sido aplicadas en Instituto y universidades. La revisión de la literatura académica permitió establecer coincidencias en los temas: dificultades de la gestión directiva durante la pandemia del COVID-19, limitaciones de gestión directiva, carencias de habilidades digitales y afecciones emocionales.

\section{DESARROLLO Y DISCUSIÓN}

De acuerdo a la revisión de la literatura académica permitió establecer tópicos en los cuales coincidieron los autores con respecto al desempeño de los directivos desde la gestión y las dificultades que afrontaron los padres de familia como parte de la comunidad, en el contexto de la emergencia sanitaria.

\section{Limitaciones de la gestión directiva}

El director debe como gestor, al asumir el liderazgo de una institución educativa contar con cualidades, que fortalezcan sus competencias en el manejo de personal, administración, pedagógicos y recursos financieros (Rojas et al., 2020) en tal sentido esa recomendable exista una profesionalización de la carrera directiva (Rodríguez, 2018). En Perú el MINEDU, ha implementado acciones positivas para favorecer el buen desempeño de los directivos, mediante capacitaciones, acompañamiento e incluso a colocado a su disposición una plataforma con todos los recursos necesarios para ejecutar su gestión; sin embargo, la profesionalización del adecuado desempeño directivo una vez que ocupa su cargo corre por su cuenta, de Diplomados o talleres, que orienten su formación profesional para la mejora de la calidad en las instituciones educativas que dirigen. Además, es importante precisar que la división de las escuelas que existen en Perú resulta compleja para atender a todos los colegios por igual, desde el MINEDU se prioriza a las instituciones educativas de gestión pública, mientras que la iniciativa de ayuda a las escuelas privadas depende de las Unidades de Gestión Local (UGEL). 
Al estallar la emergencia sanitaria, se brindaron normativas para mejorar el servicio educativo en instituciones públicas como privadas, sin embargo, se reguló el sistema de trabajo para los colegios estatales, como fue la RV $\mathrm{N}^{\circ} 155$ "Disposiciones para el trabajo de los profesores y auxiliares de educación que aseguren el desarrollo del servicio educativo de las instituciones y programas educativos públicos, frente al brote del COVID-19" (MINEDU, 2021a) mientras que para las escuelas privadas se desarrollaron normativas específicas de sincerar los costos, llegara acuerdo con los padres sobre el costo de las pensiones e informar cómo se ejecutaría la educación virtual, esto generó conflicto con los padres (Gonzales-Sánchez et al., 2021), dificultades que también demostró el informe de la Defensoría del Pueblo (2020) sobre los principales cuestionamientos de los padres de familia al implementarse la educación a distancia o remota acerca de que si estas instituciones podían implementa una educación de calidad (GonzalesSánchez et al., 2021).

La pandemia, exigió poner en práctica actitudes que permitieran acercar a la comunidad educativa, para Aparico et al., (2020) "el éxito escolar en contextos desfavorecidos implica asumir los principios de inclusión, identidad y de justicia social para atender las necesidades singulares de esos contextos" (p. 187). En esta misma línea Moswela y Kgosidialwa (2019) señalaron que, el liderazgo directivo siempre enfrenta obstáculos, por tal razón, se debió atender a todos los estudiantes por igual buscando acercamiento con las familias dentro del trabajo comunitario, los padres se convertirán en aliados estratégicos para permitir que la educación no se detenga.

\section{Las carencias de habilidades digitales}

La pandemia develó múltiples dificultades en la escuela peruana, siendo la más significativa el adecuado uso de las TIC, tanto por docentes, estudiantes y padres de familia, al determinarse que los medios de comunicación fuera sincrónicos o asincrónicos, significó involucrar a todos en estos significados, lo acelerado como se dieron los acontecimientos, hizo que se ensañaran formas de su uso de acuerdo a cada escuela, para realizar la inducción en cada comunidad educativa; el principal limitante fue el acceso a conexión de internet. En las escuelas públicas se presentó un importante número de estudiantes que abandonaron las clases, por esta razón haciendo uso de los aliados como Comisaría, Demuda, Municipios, entre otras redes institucionales, tuvieron que buscar a los estudiantes para que retornaran a sus clases, desde el gobierno central fueron distribuidas dispositivos electrónicos para que los estudiantes de menor acceso pudiesen tener una mejor conexión; sin embargo, el tema de fondo fue la brecha digital, las carencias en contar con conexión a internet, que afectó no solo a los estudiantes sino también a los docentes (Gómez y Escobar, 2021).

En esta línea Owolabi (2020) quien estudio el tema en África, sostuvo que la educación virtual debió buscar oportunidades para que el acceso fuese equitativo, poniendo a disposición de los estudiantes y docentes los recursos necesarios para su desarrollo académico, además, la eficiencia ya no estar de acuerdo al rendimiento académicos; sino al desenvolvimiento delos estudiantes en este contexto, similar situación se evidenció principalmente en escuelas públicas de México Portillo et al., (2020) determinar que, este sistema educativo incrementó 
las desigualdades. Para Dong et al., (2020), quienes estudiaron este tema en China, describieron que los padres antes de la pandemia veían con agrado la implementación de algunas experiencias que permitirán la educación digital; sin embargo el acelerado suceso que determinó la pandemia del COVID-19, representó una situación desafiante, en la cual también si se darían aprendizajes pertinentes de calidad. En Estados Unidos Price et al., (2021) sostuvieron que estos fueron momentos complejos en los cuales fue común ver a las docentes ir con carteles a buscar a sus alumnos, los padres tuvieron la necesidad de comprar dispositivos tecnológicos para que ellos pudiera llevar las clases virtuales.

\section{Afecciones emocionales}

En Perú el tema emocional tomó considerable relevancia durante la pandemia, los hogares se convirtieron en escuelas y los padres fueron los responsables en ayudar a sus hijos; sin embargo la educación ha cambiado mucho en Perú de acuerdo a como se les fue impartida a los mayores, antes se valoraba los aspectos cognitivos o memorísticos, sin embargo, las recomendaciones curriculares se promueve el constructivismo, trabajo cooperativo y la autonomía; por tal razón resultó difícil atender a los niños e casa (Janampa et al., 2021), además en informes emitidos por la Defensoría del Pueblo, lanzó la alerta de que se estaban incrementando los casos de violencia familia, de agresiones a los estudiantes (Sánchez Boris, 2021).

Similar situación afrontaron los padres de distintos lugares del mundo, en Italia para Cusinato et al., (2020) el aislamiento provocó en los menores ansiedad y angustia, coincidencia con el estudio de (Jeffs et al., 2021) en Nueva Zelanda, el poco contacto con otros compañeros se manifestó con diversos síntomas como la pérdida del sueño, al igual (Misirli y Ergulec, 2021) en Turquía señalaron que, las implicancias psicológicas para los niños fueron significativas, no hay duda de las implicaciones a futuro de las afecciones psicológicas e los alumnos; a pesar del retorno a clases en gran parte de los países del mundo incluido Perú en el año 2021, la educación no es igual, debido a la necesidad de proteger su salud, tomando medidas de distanciamiento y priorizando contenidos curriculares.

Con respecto al tema emocional en los padres de familia, las medidas restrictivas que se dieron al iniciar la pandemia, les dieron tranquilidad, la novedad que el COVID-19, representaba y las implicancias en la salud, afloraron temores justificados de enfermarse ellos o sus hijos; sin embargo, conforme transcurrió la cuarentena el encierro generó múltiples dificultades las cuales incrementaron sus condiciones de estrés, como fueron las pérdidas de sus trabajos, trajo consigo apuros económicos e las familias, otros trasladaron su trabajo a casa limitando sus tiempos. En Australia Westrupp et al., (2021) señalaron que, las familias se sintieron vulnerables por las dificultades económicas, incrementando las condiciones de estrés, coincidieron con Toran et al., (2021) este contexto incrementó la ansiedad, agresividad e irritabilidad, de los padres en el estudio comparativo que realizaron de China y Turquía.

La regulación de emociones fue un tema familiar complicado durante la emergencia sanitaria, en tal sentido desde la gestión directiva debieron tenderse formas de acercar a los padres con la escuela, por tal motivo los directivos, tuvieron que realizar 
todos los esfuerzos para permitir la ejecución de la nueva normalidad educativa y poner en práctica la escucha activa y comunicación asertiva.

\section{CONCLUSIÓN}

Tomando en cuenta el objetivo que se formuló explorar desde la literatura académica la gestión directiva y el trabajo con la comunidad durante la emergencia sanitaria por el COVID-19, se concluyó que en Perú los directores, afrontaron dificultades de poder involucrar a su comunidad educativa al sistema educativo, porque fue tan repentino la implementación por las autoridades debido a la pandemia para lo cual no estaban preparados, además emergieron dificultades, limitaron su ejecución, como fueron la poca conexión a Internet, desconocimiento de herramientas y estrategias para desarrollar las experiencias de aprendizaje, tanto en la conexión sincrónica o asincrónica, otro factor fue la falta de dispositivos que permitirán la conectividad, el teléfono se convirtió en el instrumento más común para comunicarse y recibir las clase.

Con respecto a la comunidad, representada por los padres de familia como parte de la comunidad afrontar severas condiciones de estrés, debido las o problemas como caer enfermo, pérdida del empleo, o desarrollar el trabajo en casa, lo que originó temas emocionales como la ansiedad, incertidumbre, irritabilidad, que lamentable te afectaron en su relación con los hijos. Los cambios en la forma de enseñanza que se brindan, también complicar la situación de poder ayudar a sus hijas por desconocimiento a los temas, debido a las diferencias generacionales. Sin embargo, no todo fue negativo porque tanto los directivos y padres, evidenciaron ser residentes, adaptándose a la nueva normalidad.
REFERENCIAS

Acevedo, C., Valenti, G., y Aguiñaga, E. (2017). Gestión institucional, involucramiento docente y de padres de familia en escuelas públicas de México. Calidad En La Educación, 46, 53-95. https://doi.org/10.4067/s071845652017000100053

Aparico, C., Sepúlveda, F., Valverde, X., Cárdenas, V., Contreras, G., y Valenzuela, M. (2020). Liderazgo directivo y cambio educativo_ Análisis de una experiencia de colaboración UniversidadEscuela.pdf. Páginas de Educación, 13, 19-41. file:///C:/Users/ADMIN/Documents/Vista de Liderazgo directivo y cambio educativo_ Análisis de una experiencia de colaboración Universidad-Escuela.pdf

Carrasco, I., Mujica, A., Bustos, C., Villalobos, M., Tamarín, C., Olave, y Medel. (2017). Valoración de la influencia de un programa de acompañamiento directivo sobre la percepción y satisfacción laboral de docentes y directivos. Universitas Psychologica, 16(2). https://doi. org/10.11144/Javeriana.upsy16-2.vipa

CEPAL-UNESCO. (2020). La educación en tiempos de la pandemia COVID-19. Comisión Económica Para América Latina y El Caribe, Santiago Oficina Regional de Educación Para América Latina y El Caribe de La Organización de Las Naciones Unidas Para La Educación La Ciencia y La Cultura, 11, 11-13. https://unesdoc.unesco. org/ark:/48223/pf0000374075?posInSet $=1 \& q u$ eryId=9ccf4a39-7c50-43e4-856b-a09632daa7a2

Cusinato, M., Lannattone, S., Spoto, A., Poli, M., Moretti, C., Gatta, M., y Miscioscia, M. (2020). Stress, resilience, and well-being in Italian children and their parents during the COVID-19 pandemic. International Journal of Environmental Research and Public Health, 17(22), 1-17. https://doi.org/10.3390/ ijerph 17228297

Defensoría del Pueblo. (2020). Educación En Pandemia. https://www.defensoria.gob.pe/ wp-content/uploads/2020/08/Serie-InformesEspeciales- $\mathrm{N}^{\circ}$-027-2020-DP-La-educaciónfrente-a-la-emergencia-sanitaria.pdf 
Dong, C., Cao, S., y Li, H. (2020). Young children's online learning during COVID-19 pandemic: Chinese parents' beliefs and attitudes. Children and Youth Services Review, 118(August), 105440. https://doi.org/10.1016/j. childyouth.2020.105440

Gómez A, y Escobar, F. (2021). Educación virtual en tiempos de pandemia: Incremento de la desigualdad social En Perú. Biblioteca Electrónica Científica En Línea, I, 1-13. https:// preprints.scielo.org/index.php/scielo/preprint/ view/1996\#.YFvtuoYYVe0.mendeley

Gonzales-Sánchez, A., Berrío-Quispe, M., Alegría, R., y Trujillo, M. (2021). Management and the Promotion of Educational Quality During the Health Emergency. Revista Gestão Inovação e Tecnologias, 11(3), 649-660. https://doi. org/10.47059/revistageintec.v11i3.1965

Gonzales-Sánchez, A., Trujillo, N., Pelayo, I., Ocaña-Fernández, Y. (2021). Management in strengthening social- emotional competence during health emergencies: Gestão no fortalecimento da competência socioemocional durante emergências de saúde. Revista Tempos e Espaços Em Educação, 14(33), e15111. https:// doi.org/10.20952/revtee.v14i33.15111

Janampa, E., Gonzales-Sánchez, A., Bayona, G., y Valqui, J. (2021). Acompanhamento familiar aos alunos durante a pandemia COVID-19.pdf. Laplage Em Revista. https://doi.org/https://doi. org/10.24115/S2446-6220202173C1502p.43-50

Jeffs, E., Lucas, N., y Walls, T. (2021). CoVID-19: Parent and caregiver concerns about reopening New Zealand schools. Journal of Paediatrics and Child Health, 57(3), 403-408. https://doi. org/10.1111/jpc.15234

López, E., García, L., y Martínez, J. (2019). La gestión directiva como potenciadora de la mejora del clima organizacional y la convivencia en las instituciones de educación media superior. RIDE Revista Iberoamericana Para La Investigación y El Desarrollo Educativo, 9(18), 792-812. https:// doi.org/10.23913/ride.v9i18.471

Lozano-Vargas, A. (2020). Impacto de la epidemia del Coronavirus (COVID-19) en la salud mental del personal de salud y en la población general de China. Revista de Neuropsiquiatría, 83(1), 51-56. https://doi.org/10.20453/rnp.v83i1.3687

MINEDU. (2020). Nº93 RV Aprobar el documento normativo denominado "Orientaciones pedagógicas para el servicio educativo de Educación Básica durante el año 2020 en el marco de la emergencia sanitaria por el Coronavirus COVID-19”. https://www.gob.pe/institucion/ MINEDU/normas-legales/535987-093-2020MINEDU

MINEDU. (2021a). "Disposiciones para el trabajo de los profesores y auxiliares de educación que aseguren el desarrollo del servicio educativo de las instituciones y programas educativos públicos, frente al brote del COVID-19." 180

MINEDU. (2021b). Guía para la gestión escolar en II.EE. y programas educativos de Educación Básica

MINEDU. (2014). Marco de buen desempeño del directivo. 56. http://www. MINEDU.gob.pe/ DeInteres/xtras/marco_buen_desempeno_ directivo.pdf

Misirli, O., y Ergulec, F. (2021). Emergency remote teaching during the COVID-19 pandemic: Parents experiences and perspectives. Education and Information Technologies, 0123456789. https://doi.org/10.1007/s10639-021-10520-4

Moswela, B., y Kgosidialwa, K. (2019). Leadership and school success: Barriers to leadership in Botswana primary and secondary schools. Educational Management Administration and Leadership, 47(3), 443-456. https://doi. org/10.1177/1741143217739355

Owolabi, J. (2020). Virtualising the school during covid-19 and beyond in africa: Infrastructure, pedagogy, resources, assessment, quality assurance, student support system, technology, culture and best practices. Advances in Medical Education and Practice, 11, 755-759. https://doi. org/10.2147/AMEP.S272205

Pérez, A. (2014). Enfoque de la gestión escolar: una aproximación desde el contexto latinoamericano. Educación y Educadores, 17(2), 357-369. https:// doi.org/10.5294/edu.2014.17.2.9 
Portillo, S., Castellanos, L., Reynoso, O., y Gavotto, O. (2020). Enseñanza remota de emergencia ante la pandemia Covid-19 en Educación Media Superior y Educación Superior [Emergency remote teaching in the face of the Covid-19 pandemic in Higher Secondary Education and Higher Education]. Propósitos y Representaciones, 8(3), 1-17. https://n9.cl/szvv0

Price, D., Peersman, J., y Matherne, S. (2021). It's Not Homeschool, It's School at Home: Parents' Experiences as Teachers during the COVID-19 Pandemic. Educational Media International, 58(2), 102-123. https://doi.org/10.1080/095239 87.2021.1930486

Rodríguez, E. (2018). El ejercicio de la función directiva en contextos complejos: su profesionalización. Perspectiva Educacional, 57(3), 131-152. https://doi. org/10.4151/07189729-vol.57-iss.3-art.775

Rojas, O., Martínez, M., y Riffo, R. (2020). Gestão diretiva e estresse laboral do profissional docente: um olhar a partir da pandemia COVID-19. In Revista on line de Política e Gestão Educacional (pp. 1226-1241). https://doi.org/10.22633/rpge. v24i3.14360
Sánchez Boris, I. M. (2021). Impacto psicológico de la COVID-19 en niños y adolescentes. Medisan, 25(1), 123-141.

Toran, M., Sak, R., Xu, Y., Şahin-Sak, İ. T., y Yu, Y. (2021). Parents and children during the COVID-19 quarantine process: Experiences from Turkey and China. Journal of Early Childhood Research, 19(1), 21-39. https://doi. org/10.1177/1476718X20977583

Westrupp, E., Stokes, M., Fuller-Tyszkiewicz, M., Berkowitz, T., Capic, T., Khor, S., Greenwood, C. J., Mikocka-Walus, A., Sciberras, E., Youssef, G. J., Olsson, C. A., y Hutchinson, D. (2021). Subjective wellbeing in parents during the COVID-19 pandemic in Australia. Journal of Psychosomatic Research, 145, 110482. https:// doi.org/10.1016/J.JPSYCHORES.2021.110482 